\title{
Emergency Management of Ischemic Stroke in Children
}

\author{
Maja Steinlin, $M D^{1,2, *}$ \\ Mark T. Mackay, MBBS ${ }^{3,4}$
}

\author{
Address \\ *,1Paediatric Neurology, University Children's Hospital and Neurocentre, \\ Inselspital Bern, Bern, 3010, Switzerland \\ Email: maja.steinlin@insel.ch \\ ${ }^{2}$ University of Bern, Bern, 3012, Switzerland \\ ${ }^{3}$ Department of Neurology, The Royal Children's Hospital Melbourne, 3 West \\ Clinical Offices Room 3.062, 50 Flemington Road, Parkville, Melbourne, Victoria \\ 3052, Australia \\ Email:mark.mackay@rch.org.au \\ ${ }^{4}$ Murdoch Children's Research Institute, University of Melbourne, Parkville, Mel- \\ bourne, Australia
}

Published online: 9 April 2015

(C) Springer Science+Business Media New York 2015

This article is part of the Topical Collection on Cerebrovascular Disorders

Keywords Ischaemic stroke - Children - Emergency - Recognition tools - Chain of survival - Diagnostic workup • Emergency treatment - Emergency workup - Thrombolysis - Aspirin • Heparin - Decompressive craniotomy · Supportive therapy · Steroids · Neuroimaging

\section{Opinion statement}

Children who present with acute neurological symptoms suggestive of a stroke need immediate clinical assessment and urgent neuroimaging to confirm diagnosis. Magnetic resonance imaging (MRI) is the investigation of first choice due to limited sensitivity of computed tomography (CT) for detection of ischaemia. Acute monitoring should include monitoring of blood pressure and body temperature, and neurological observations. Surveillance in a paediatric high dependency or intensive care unit and neurosurgical consultation are mandatory in children with large infarcts at risk of developing malignant oedema or haemorrhagic transformation. Thrombolysis and/or endovascular treatment, whilst not currently approved for use in children, may be considered when stroke diagnosis is confirmed within 4.5 to $6 \mathrm{~h}$, provided there are no contraindications on standard adult criteria. Standard treatment consists of aspirin, but anticoagulation therapy is frequently prescribed in stroke due to cardiac disease and extracranial dissection. Steroids and immunosuppression have a definite place in children with proven vasculitis, but their role in focal arteriopathies is less clear. Decompressive craniotomy should be considered in children with deteriorating consciousness or signs of raised intracranial pressure. 


\section{Introduction}

Over the last three decades, tremendous improvement has been achieved in emergency treatment of adult stroke with faster recognition, decreasing time to treatment and new treatment options. Systems of care which include predefined prehospital and emergency department protocols and diagnostic algorithms [1] have increased access to hyperacute interventions which improve outcomes by salvaging viable brain [2]. Unfortunately, children are not receiving the benefits of these treatments due to inordinate delays related to poor recognition of stroke symptoms and limited access to emergent imaging $[3,4,5 \bullet \bullet, 6,7]$.

Although childhood stroke has gained increasing attention, little still is known on treatment in general and especially emergency treatment of children with stroke. Existing guidelines $[8,9 \bullet, 10 \bullet]$ rely largely on expert opinions and extrapolation of adult evidence. However, it is well established that childhood stroke differs in aetiology [11-15]; thus, treatment should be tailored to specific paediatric needs.

This review will provide an overview of the importance of clinical recognition of childhood stroke by paramedics and emergency staff, compare adult bedside stroke recognition tools and discuss their suitability in children. In a second part, the stroke chain of survival focusing on important components of the emergency assessment of childhood stroke will be discussed. Last but not least, current knowledge and evidence on acute stroke treatments in children will be reviewed.

\section{Clinical recognition of childhood stroke}

\section{Symptoms and signs of stroke}

Most symptoms and signs in childhood stroke are similar to those of adult stroke with the exception of seizures. Prospective population-based studies suggest similar frequencies of headache, hemiparesis and facial weakness [14-17] but increased frequency of speech disturbance and seizures when compared to adults (Table 1). The frequent occurrence of seizures in childhood stroke is a notable difference to adults, occurring in up to $50 \%$

\begin{tabular}{|c|c|c|c|c|}
\hline & $\begin{array}{l}\text { Adults in \% } \\
\text { Rathore et } \\
\text { al. [16] }\end{array}$ & $\begin{array}{l}\text { CH Children in \% } \\
\text { Steinlin et al. } \\
\text { [15]/updated }\end{array}$ & $\begin{array}{l}\text { AU children in \% Yock- } \\
\text { Corrales et al. }[17,30]\end{array}$ & $\begin{array}{l}\text { GB children in } \% \\
\text { Mallick et al. }[5,14]\end{array}$ \\
\hline Headache & 22 & 28 & 46 & 24 \\
\hline Vertigo & 3 & 3 & ?? & ?? \\
\hline Seizures & 3 & 26 & 17 & 29 \\
\hline Speech problems & 26 & 41 & 46 & 46 \\
\hline (Hemi)paresis & 87 & 79 & $57-63$ & 72 \\
\hline Facial palsy & 58 & 40 & 46 & 41 \\
\hline Visual problems ${ }^{a}$ & 17 & 14 & 19 & 5 \\
\hline Ataxia & ?? & 13 & 10 & ?? \\
\hline Sensory problems & 44 & 16 & 25 & ?? \\
\hline
\end{tabular}


of cases [14, 18-20]. Particular challenges to diagnosis include (a) the greater difficulty identifying posterior circulation strokes because they are often associated with non-specific signs such as headaches, nausea and vomiting, (b) children having difficulty articulating their symptoms and (c) clinical assessment being sometimes affected by limited ability to cooperate with formal neurological examination (especially important for evidence of ataxia, mild facial palsy and visual problems).

\section{Differential diagnosis of stroke in children}

Stroke is the cause of focal neurological symptoms in approximately three quarters of adults. In contrast, there is a low a priori probability of stroke in

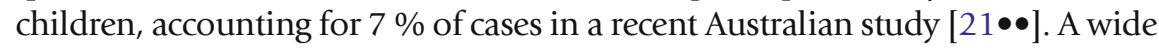
variety of conditions mimic stroke $[21 \bullet \bullet, 22,23]$ which differ from adults.

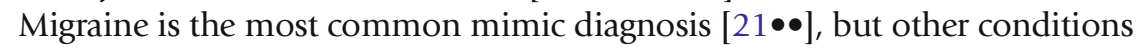
include seizures, posterior reversible leucoencephalopathy, intracranial infections, demyelinating disorders, metabolic stroke, tumours, drug toxicity, conversion disorder problems and syncope.

Knowledge of stroke symptoms in the community, amongst paramedics, primary care and emergency physicians, is essential to decreasing diagnostic delays. Public education campaigns such as Face-Arm-Speech-Time (FAST) and SUDDENS [the five sudden stroke warning signs: 1) weakness or numbness, 2) troubles talking or confusion, 3) troubles seeing, 4) troubles walking, imbalance or dizziness; 5) sudden headache] have been developed to improve lay awareness of stroke. The FAST message [24] relies on three symptoms: facial palsy, hemiparesis and speech problems. FAST is less sensitive than the SUDDENS message capturing additional symptoms of severe headache, focal numbness, visual disturbance, dizziness, trouble walking, loss of balance or coordination. SUDDENS is more sensitive than FAST but more difficult to remember [25]. Therefore, many countries around have adopted the simpler FAST message.

\section{Bedside stroke recognition tools}

Paramedics, triage nurses and emergency physicians are the first point for many patients with stroke [26], and bedside stroke recognition tools have been developed to assist these health professionals to identify stroke. Paramedic tools include the most widely used FAST [24] and the Cincinnati Prehospital Stroke Scale (CPSS) [27] - both shown to have the best operating characteristics in a systematic review [28]. Emergency physician tools include the Recognition of Stroke in the Emergency Room (ROSIER) tool [29]. Most tools include "absence of seizures" as a variable to increase specificity by identifying mimics. Thus, these tools may have limited utility in children because seizures are a frequent presenting symptom of stroke. A comparison of FAST and ROSIER in a paediatric emergency setting revealed both tests to have reasonable overall sensitivity (78 and $81 \%$,

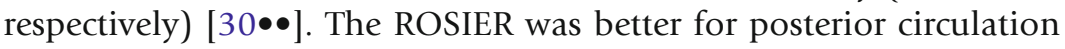
stroke, which accounts for approximately one third of all events [31, 32]. 
Specificity of bedside tools needs to be determined in prospective paediatric cohorts.

\section{The Stroke Chain of Survival: improving access to hyperacute therapies}

There is a major focus in adults on reducing delay to stroke diagnosis because thrombolysis is established as an effective treatment if given up to $6 \mathrm{~h}$ from symptom onset [2]. The Stroke Chain of Survival and Recovery is a framework devised by the National Institute of Neurological Disorders and Stroke (NINDS) to improve acute stroke care by identifying key points at which delays occur which can be targeted by interventions $[33,34]$. Adult emergency stroke management guidelines recommend radiological confirmation of diagnosis within $45 \mathrm{~min}$, decision to thrombolyse within $60 \mathrm{~min}$ and admission to a stroke unit within $3 \mathrm{~h}[33,34]$.

Understanding of reasons for delay along the paediatric Stroke Chain of Survival, from symptom onset to radiological diagnosis, is essential to improve outcomes for children affected by stroke. Prehospital factors, such as failure to call an ambulance, are important contributors to delayed diagnosis in adults [35]. In contrast, in-hospital factors contribute more to delayed diagnosis in children. Limited access to diagnostic imaging is probably the biggest obstacle

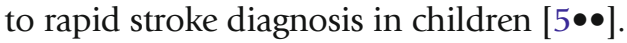

Potential interventions in the prehospital setting include the parental education, particularly for children affected by congenital heart disorders or sickle cell disease who are at increased risk of stroke. Development of primary paediatric stroke centres with well-developed diagnostic and acute management protocols will increase access to hyperacute therapies [36].

\section{Assessment and diagnosis of stroke}

\section{Clinical evaluation of children with suspected stroke}

Similar to adults, predefined pathways adjusted to different local in-hospital facilities need to be in place. Primary survey should include assessment of vital signs, including blood pressure and saturations, and level of consciousness. Assessment of symptom severity should be incorporated into the neurological examination. The NIH stroke scale which has been recently validated in children pedNIH scale [37] provides an overall impression of the severity of the clinical situation, and can be used to monitor clinical course. Stuttering and worsening of symptoms are frequent in childhood-onset stroke and may be a clue to specific aetiologies such as focal cerebral arteriopathy [38]. In contrast, embolic strokes typically reach maximum severity immediately following symptom onset.

Once a child has arrived in emergency, immediate investigations are required to confirm the clinical suspicion of stroke and initiate treatment. CT imaging to identify intracranial haemorrhage prior to consideration of thrombolysis is the standard adult approach to stroke diagnosis. MRI is the 
investigation of first choice in children with suspected ischaemic stroke for several reasons (Fig. 1). The low probability of stroke in children means it has to be positively diagnosed on imaging $[21 \bullet \bullet, 22,23]$. CT imaging has poor sensitivity for detection of acute ischaemia, ranging from 16 to $56 \%$ in paediatric studies $[6,7,39,17,5 \bullet \bullet]$. As summarized in Fig. 1, MRI has also the potential to answer further important questions concerning vessel status [40], diffusion-perfusion mismatch (important for decision on lyses) [41], search for dissections [42•] and inflammatory signs [43]. Equally important is that MRI is more sensitive for detection of some mimic diagnoses including demyelinating disorders, encephalitis and tumours, particularly those involving the posterior fossa. Some of this information can be obtained by modern CT scanners [44], but there are concerns about the lifelong risk of malignancies with exposure to CT imaging in children $[45,46]$. Accessing MRI imaging in the emergency setting is challenging, as

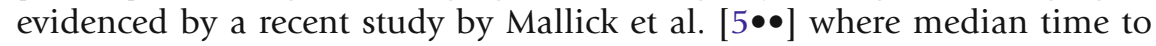
confirmation of stroke diagnosis was $23 \mathrm{~h}$. However, recent adult studies have demonstrated the feasibility of rapid MRI protocols in the ED [47•].

\section{Laboratory investigations}

Basic blood work in the emergency department should include full blood count, electrolytes, glucose and renal and liver function. Further investigations as summarized in Table 2 might be important to initiate immediately after diagnosis (as signs for infectious or inflammatory aetiology); others can/have to be done at a later stage (as prothrombotic disorders). There is increasing data to support the importance of CSF analysis in childhood stroke [48], although it is contraindicated in children requiring anticoagulation treatment. Indicators of infectious or inflammatory processes include elevated opening pressure, pleocytosis and elevated protein [49].

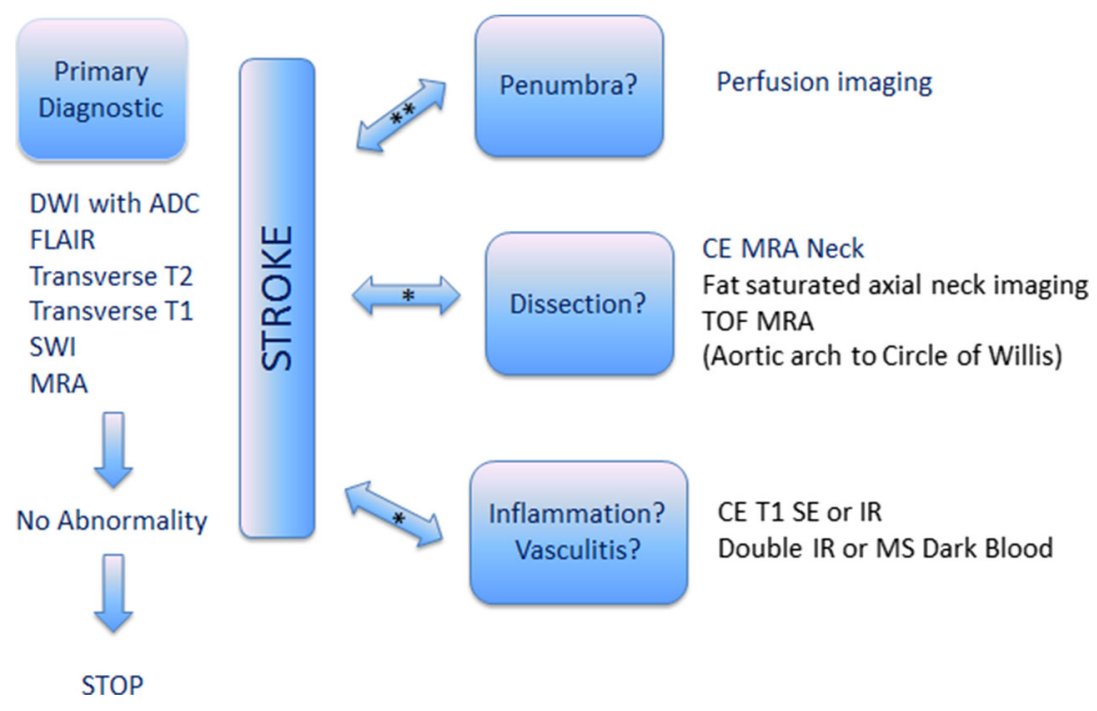

Fig. 1. Neuroimaging for children with suspected stroke. DWI diffusion weighted images, $A D C$ apparent diffusion coefficient, CE contrast enhanced, MRA magnetic resonance angiography, SWI susceptibility weighted images, TOF time of flight, SE spin echo, $I R$ inversion recovery. * May be done in a second examination within the first few days. ${ }^{* *}$ In case of considering thrombolysis.

Inflammation? CE T1 SE or IR Double IR or MS Dark Blood 


\section{Table 2. Suggested laboratory investigations}

\begin{tabular}{|c|c|c|c|}
\hline & Results as an emergency & To do at diagnosis & To do at appropriate time \\
\hline Blood & $\begin{array}{l}\text { BC, SR, CRP, electrolytes, } \\
\text { glucose, liver and renal } \\
\text { function, coagulation, } \\
\text { lactate }\end{array}$ & $\begin{array}{l}\text { Viral serology (as varicella } \\
\text { /herpes simplex/Mycoplasma } \\
\text { /Enterovirus), borreliosis, } \\
\text { vasculitis screen (ESR, ACLA, } \\
\text { ANA, LA) }\end{array}$ & $\begin{array}{l}\text { Lipid profile, homocystein, } \\
\text { prothrombotic studies } \\
\text { (factor V Leiden, prothrombin } \\
\text { and MTHRF mutation, lipoprotein A, protein } \\
\text { S and C), specific investigations }\end{array}$ \\
\hline Urine & & Organic acids ${ }^{a}$, amino acids $^{a}$ & \\
\hline CSF & $\begin{array}{l}\text { Opening pressure, cells, } \\
\text { protein, glucose, lactate }\end{array}$ & $\begin{array}{l}\text { PCR varicella, other PCR/ } \\
\text { serologies }\end{array}$ & \\
\hline
\end{tabular}

\section{Acute treatment of stroke}

Treatment recommendations in published paediatric stroke guidelines are largely based on expert consensus opinion or extrapolation from adult data due to a lack of evidence from randomized controlled trials $[8,10 \bullet]$.

General principles of stroke care

In any child with stroke, it is important to secure the basic $\mathrm{ABC}$ of airway, breathing and circulation. In contrast to children with haemorrhagic stroke, a significant reduction in level of consciousness is unusual in children with ischaemic stroke $[14,17]$. There is conflicting evidence to support oxygen therapy by mask breathing in adults with normal saturations ( $\geq 92 \%)[50,51]$. No such treatment seems to be justified in children, in the absence of significant cardiac disease, but careful monitoring and maintaining of oxygen saturations $\geq 92 \%$ is important and recommended in adult [1] and paediatric [8] guidelines. Arterial hypertension is a major risk factor for stroke in adults, and many adults have elevated blood pressure at presentation [52]. Elevated blood pressure and rapid reduction of blood pressure both have negative effects on outcome. In view of this equipoise, adult stroke guidelines propose an optimal blood pressure to be in the mild to moderately elevated range, based on data from randomized controlled trials $[1,53]$. Elevated blood pressure is reported in approximately two thirds of children in the first $24 \mathrm{~h}$ following stroke onset, and in about $20 \%$ during the first 3 days, mainly in children with cardiac problems, Moyamoya and severe occlusive vasculopathy [54, 55]. It is possible that the elevated blood pressure may be a compensatory phenomenon to maintain cerebral perfusion, but increasing hypertension may also be the first sign of raised intracranial pressure. Elevated blood pressure in the first few days should result in assessment for malignant cerebral oedema, haemorrhagic conversion and cardiac or renovascular disease. Correction of blood pressure should be done cautiously in line with adult recommendations. Maintenance of euvolemia in patients with stroke is important, for adults as well as for 
children. Hypovolemia might result in hypoperfusion and thrombocytosis, and promote thrombosis. In children at risk of malignant swelling of infarction, careful monitoring of fluid balance is necessary and, for intravenous fluid, isotonic solution is important. Pyrexia is associated with increased short-term mortality in adults [56]. Paracetamol is recommended for adults with fever [57]. A similar approach is recommended for children even though no specific paediatric data are available [8]. For pre-schoolers, treatment for fever may also reduce the risk of acute symptomatic seizures. It makes common sense to treat infectious foci by specific antiinfectious agents. For adults, hypo- and hyperglycaemia should be avoided [58, 59], because persistent hyperglycemia leads to increase of infarct volume and cerebral oedema, both negatively affecting outcome. Significant hypoglycaemia is known to provoke seizures. Therefore, blood sugar monitoring is recommended in the acute phase following stroke onset. Without accompanying diabetic or metabolic disorders, glucose problems in children are rare.

Despite lack of any evidence, there is in large a basic agreement on how to treat

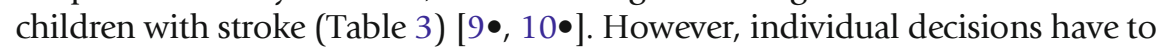
be made carefully, considering not only known evidence in adults but also the special aspects in each child.

\section{Thrombolysis and mechanical thrombectomy}

The ischaemic penumbra, which surrounds the infarct core, is the target for hyperacute interventions. This hypoperfused tissue, which is functionally inactive but structurally intact, can be salvaged with recanalization. The time characteristics of the penumbra have not been adequately studied in children.

However collateralization, which has an important influence on infarct growth, is thought to be superior in children $[60-62,41]$ and may mean there is a longer time window for intervention.

\section{Table 3. Medical treatment options}

\begin{tabular}{|c|c|c|}
\hline & & Caveats \\
\hline Aspirin & $\begin{array}{l}\text { Baseline treatment } \\
5 \mathrm{mg} / \mathrm{kq} \mathrm{BW}\end{array}$ & $\mathrm{r} / \mathrm{o}$ first dissection and cardiac problem \\
\hline Heparinoids $^{a}$ & $\begin{array}{l}\text { Extracranial dissection } \\
\text { Cardioembolic stroke }\end{array}$ & $\begin{array}{l}\text { Caution required with large hemispheric } \\
\text { or posterior fossa infarction }\end{array}$ \\
\hline $\begin{array}{l}\text { Thrombolysis } \\
\text { Intravenous/intra-arterial }\end{array}$ & $\begin{array}{l}\text { Considering in case of vessel occlusion } \\
\text { and DW/perfusion mismatch } \\
\text { pedNIH }>>4 \text {; within a } 4.5 \mathrm{~h} / 6 \mathrm{~h} \text { time window }\end{array}$ & $\begin{array}{l}\text { Evidence limited to uncontrolled case reports, } \\
\text { often not conforming to adult guidelines }\end{array}$ \\
\hline Thrombectomy & $\begin{array}{l}\text { Considering in case of large-vessel occlusion } \\
\text { and diffusion/perfusion mismatch } \\
\text { pedNIH }>>4 \text {; within recommended adult } \\
\text { time windows }\end{array}$ & Evidence limited to uncontrolled case reports \\
\hline Steroids & $\begin{array}{l}\text { Focal (transient) arteriopathy } \\
\text { Medium- to large-vessel vasculitis }\end{array}$ & No evidence \\
\hline Immunosuppression & Small-vessel vasculitis & \\
\hline
\end{tabular}


In adults, intravenous thrombolysis and intra-arterial lyses improve outcome if initiated within time limits of 3.5-4 and $6 \mathrm{~h}$, respectively [63-65]. Time to recanalization is one of the important predictors of outcome [41]. Recanalization is more often achieved by intra-arterial thrombolysis than intravenous thrombolysis [66]. For these reasons, bridging intravenous thrombolysis,

followed by angiography and additional intra-arterial thrombolysis, is becoming the treatment of choice in adults [67]. There are increasing data to support expanding the treatment time window and offering treatment to elderly patients [68]. The recently published study MR CLEAN has shown that mechanical thrombectomy is superior to lyses by tpA or urokinase [69].

There are no controlled data to support thrombolysis or thrombectomy in children. But there are increasing reports of off-label usage of tPA in children. In a review of the literature summarizing 17 cases after thrombolysis (iv or ia), outcome was successful in $71 \%$ [70]. In another study of 9257 children, only $0.7 \%$ received thrombolysis [71]. A review of centres contributing data to the International Stroke Registry [72] found that $2 \%$ of 687 children received tPA but treatment often did not comply with recommended adult guidelines. Time to administration ranged from 2 to $52 \mathrm{~h}$ for intravenous and 3.8 to $24 \mathrm{~h}$ for intra-arterial tPA. Symptomatic intracranial haemorrhage occurred in $26 \%$ of patients, which is much higher than the $7 \%$ bleeding rates reported in adult trials [2]. Outcome of these children was not favourable, with two children dying and 12/15 survivors having residual neurological deficits [72]. When results were compared to ten cases previously described in the literature, children in the registry were younger $(p=0.07)$, treatment was more often delayed ( $p=0.057)$ and outcome was poorer, suggesting a previous publication bias towards cases with shorter treatment lag and better outcomes.

The most appropriate dose of tPA in children is unknown. Developmental differences in the fibrinolytic system such as lower plasminogen and free tissue plasminogen levels and higher plasminogen activator inhibitor-1 (PAI-1) levels may mean that increased tPA doses are required relative to adults to promote clot lysis [73, 74]. There may also be age-related differences in risk of bleeding complications. For example, a recent thrombolysis study in young adults aged 16 to 49 years found lower complication rates when compared to major RCTs. In particular, none of the 48 young adults treated developed symptomatic intracranial haemorrhage [75•].

Emerging evidence in adults suggest mechanical thrombectomy to be the most effective means of achieving recanalization in adults [69]. A recent literature review $[76 \bullet \bullet]$ summarized endovascular approach in 34 children. Despite delayed treatment (mean $14 \mathrm{~h}$, range 2-18 h) partial or complete recanalization was achieved in $63 \%$. Periprocedural complications were present in $29 \%$, but symptomatic haemorrhages occurred in only one child. The role of mechanical thrombectomy remains unclear, and specific challenges in children include small vessel size and frequent inflammatory reaction within the vessel wall.

In summary, there are no controlled data to support thrombolysis or mechanical thrombectomy in children with ischemic stroke. However, there is probably a subgroup of children who may benefit but treatment should be considered very cautiously and only be performed in primary stroke centres experienced in acute management of children affected by stroke [36]. In the

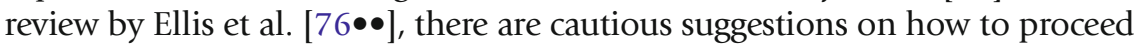
for thrombolysis/thrombectomy in children. 


\section{Aspirin versus heparin: an ongoing debate}

In children where thrombolysis is not indicated, antithrombotic treatment should be initiated immediately following confirmation of diagnosis. In adults, evidence from large randomized controlled trials supports use of aspirin over heparinoids to improve outcome and reduce early recurrence without increasing risk of early haemorrhagic complications [77]. Recent AHA guidelines suggest aspirin as first-line treatment, if lyses or endovascular approach is not indicated [1]. For children, no such trials exist. A theoretical debate on antiinflammatory and antithrombotic effects of acetylsalicylic acid versus heparinoids would suggest to favour the second. An international analysis of current practice found that aspirin, anticoagulation and no treatment were being used equally, with a trend to platelet aggregation in Europe and to anticoagulation in North America [78]. Low molecular weight heparin and clopidogrel are being increasingly used in children and are shown to be safe [79-82]. In the absence of data from randomized controlled trials, there is nonconsensus amongst experts, with one treatment guideline suggesting it is reasonable to treat initially with heparinoids until exclusion of cardioembolic cause and extracranial dissection [9•], whereas the other recommends either heparinoids or aspirin as initial therapy [10•]. For adults, it has been shown that anticoagulation is superior to aspirin for reduction of recurrent cardioembolic stroke [83]. However, a single randomized controlled trial of children with complex cyanotic heart disease did not show that heparin was superior to aspirin for children for prevention of thrombotic events following palliative surgery [84]. However, both paediatric guidelines favour use of anticoagulant treatment in children if stroke is due to cardioembolism or dissection.

No randomized controlled trials exist for dissection in adults or children, but current guidelines suggest it reasonable to anticoagulate children with stroke due to extracranial dissection $[1,42 \bullet, 8,9 \bullet, 10 \bullet]$. For adults and children, there are increasing reports of endovascular approach (stenting or ballooning) in dissection problems refractory to treatment [85-87, 42•].

Large middle cerebral artery infarctions and cerebellar infarctions are at risk of swelling during the first $72 \mathrm{~h}$, increased intracranial pressure typically manifests by increased blood pressure, followed by secondary herniation and brainstem compression causing deteriorating consciousness and risk of death. In two paediatric series, $12.5 \%$ of children with middle cerebral artery strokes [88] and $11 \%$ of posterior circulation strokes [89] developed malignant cerebral oedema. Malignant middle cerebral infarction and cerebellar infarction are associated with poor outcome, and small series suggest decompressive surgery may be of benefit $[90 \bullet, 91]$, similar to what was described in adults [92, 93]. Thus, careful monitoring of patients at risk of malignant infarction in a paediatric intensive care unit with access to emergency neurosurgical treatments is mandatory.

\section{Steroids and immunosuppression in inflammatory problems}

Infection and inflammation play an important role in stroke pathogenesis, particularly in children with cerebral arteriopathy [94, 95, 80]. Recent studies $[96,97]$ have identified changes in inflammatory biomarker profiles in children 
in the acute phase following stroke, some of which may be indicators for increased risk of recurrent event. Modern neuroimaging techniques showing vessel enhancement may provide supporting evidence of inflammation in some children with focal arteriopathies [43]. Reported recurrence risk in focal arteriopathy varies from 15 to $45 \%$, possibly with the highest risk in post varicella angiopathy $[38,95,94]$. For these reasons, adjunctive immunosuppressive agents are sometimes considered as "disease-modifying" therapy in addition to antithrombotics. Steroids might alter the natural history of focal arteriopathies and decrease the risk of recurrence. Benseler et al. found there was decreased recurrence risk in children with non-progressive large to medium vasculitis, who were treated with corticosteroids and antiplatelet therapy, when compared to those treated with antiplatelet treatment alone (11 vs $30 \%$ ) [49]. Further studies are required to demonstrate a beneficial effect of corticosteroid in children with focal self-limited vasculopathy. As varicella is frequently implicated as a cause of focal cerebral arteriopathy, additional treatment with acyclovir should also be considered, especially in children with positive VZV PCR in CSF. High-dose corticosteroids, followed by other immunosuppressive agents including mycophenolate, azathioprine or cyclophosphamide, are also used in children with suspected primary CNS vasculitis, which is typically associated with small-vessel disease.

\section{Future directions}

It is vital to develop predefined institutional acute paediatric stroke management protocols [36] because they have been shown to reduce the time to neurological assessment and increase thrombolysis rates in adults [98, 99]. These protocols should include activation of a "Code Stroke" with rapid evaluation by a paediatric stroke neurology team. Support from experienced adult stroke teams is also of value. Development of thrombolysis guidelines, which conform to standard adult inclusion and exclusion criteria, is essential to select appropriate candidates and minimize risk of complications. For example, adopting standard adult criteria of a paediatric National Institutes of Health Score of $\geq 4$ is reasonable because a recent study comparing children to young adults showed similar initial stroke severity, which correlated with the severity of later deficits, thereby disproving the longstanding belief that childhood stroke is milder and has better outcome than that of adults [100•].

There is a substantial body of evidence demonstrating the positive benefits of stroke units in reducing length of hospital stay and institutional care [101] and stroke-associated morbidity and mortality when compared to care on conventional medical wards [102, 103]. It is not practical to develop geographically distinct stroke units within paediatric hospitals because stroke is uncommon, but there is no reason why the general principles of stroke unit care cannot be applied to children. The main obstacles to development of such services are the identification and support of dedicated medical leads, development of stroke emergency algorithms, funding of stroke care coordinator positions and development of standardized paediatric stroke management guidelines.

The catch cry "Time is brain" is equally applicable to children. The social and economic burden of disease for children affected by stroke and their families is 
substantial when considered over a life which may span 50-70 years. The best way to improve outcome is to minimize extent of injury by salvaging at risk brain. Therefore, there needs to be increasing focus on developing strategies to improve access to treatments which have transformed the approach to stroke care in adults.

\section{Compliance with Ethics Guidelines}

\section{Conflict of Interest}

Maja Steinlin and Mark T. Mackay declare no conflicts of interest.

Human and Animal Rights and Informed Consent

This article does not contain any studies with human or animal subjects performed by the authors.

\section{References and Recommended Reading}

Papers of particular interest, published recently, have been highlighted as being:

- Of importance

$\bullet$ Of major importance

1. Jauch EC et al. Guidelines for the early management of patients with acute ischemic stroke: a guideline for healthcare professionals from the American Heart Association/American Stroke Association. Stroke. 2013;44(3):870-947.

2. Wardlaw JM et al. Recombinant tissue plasminogen activator for acute ischaemic stroke: an updated systematic review and meta-analysis. Lancet.

2012;23:2364-72.

3. Mackay MT et al. Arterial ischemic stroke risk factors: the international pediatric stroke study. Ann Neurol. 2011;69(1):130-40.

4. Martin C et al. Delayed diagnosis of acute ischemic stroke in children-a registry-based study in Switzerland. Swiss Med Wkly. 2011;141:13281.

5.• Mallick AA et al (2014) Diagnostic delays in paediatric stroke. J Neurol Neurosurg Psychiatry. 2014 Oct 23. pii: jnnp-2014-309188 epub ahead of print.

Very recent paper which addresses the major problem of delay in diagnosis in children with suspected stroke, especially considering the problem of adequate imaging in an emergency setting.

6. Rafay MF et al. Delay to diagnosis in acute pediatric arterial ischemic stroke. Stroke. 2009;40(1):58-64.

7. Srinivasan J et al. Delayed recognition of initial stroke in children: need for increased awareness. Pediatrics. 2009; 124:227-34.
8. Clinical Royal College of Physicians, "Stroke in childhood: clinical guidelines for diagnosis, management and rehabilitation," 2004.

9. $\quad$ Roach ES et al. Management of stroke in infants and children: a scientific statement from a Special Writing Group of the American Heart Association Stroke Council and the Council on Cardiovascular Disease in the young. Stroke. 2008;39:2644-91.

This paper and the paper by Monagle P et al (Feb 2012) summarize the present guidelines for treatment in children with stroke.

10. Monagle P et al. Antithrombotic therapy in neonates and children: antithrombotic therapy and prevention of thrombosis, 9th ed: American College of Chest Physicians evidence-based clinical practice guidelines. Chest. 2012;141(2 Suppl):e737S-801S.

This paper and the paper by Roach ES et al (2012), summarize the present guidelines for treatment in children with stroke.

11. Amlie-Lefond $\mathrm{C}$ et al. Recent developments in childhood arterial ischaemic stroke. Lancet Neurol. 2008;7(5):425-35.

12. Fullerton $\mathrm{HJ}$ et al. Risk of recurrent childhood arterial ischemic stroke in a population-based cohort: the importance of cerebrovascular imaging. Pediatrics. 2007;119:495-501. 
13. Gaensan V et al. Investigation of risk factors in children with arterial ischemic stroke. Ann Neurol. 2003;53(2):167-73.

14. Mallick AA et al. Childhood arterial ischaemic stroke incidence, presenting features, and risk factors: a prospective population-based study. Lancet Neurol. 2014;13:35-43.

15. Steinlin $\mathrm{M}$ et al. The first three years of the Swiss Neuropaediatric Stroke Registry (SNPSR): a population-based study of incidence, symptoms and risk factors. Neuropediatrics. 2005;36:90-7.

16. Rathore SS et al. Characterization of incident stroke signs and symptoms: findings from the atherosclerosis risk in communities study. Stroke. 2002;33:2718-21.

17. Yock-Corrales A et al. Acute childhood arterial ischemic and hemorrhagic stroke in the emergency department. Ann Emerg Med. 2011;58:156-63.

18. Abend NS et al. Seizures as a presenting symptom of acute arterial ischemic stroke in childhood. J Clin Neurophysiol. 2013;30:134-42.

19. Hsu CJ et al. Early-onset seizures are correlated with late-onset seizures in children with arterial ischemic stroke. Stroke. 2014;45:1161-3.

20. Singh RK et al. Seizures in acute childhood stroke. J Pediatr. 2012;160:291-6.

21.• Mackay MT et al. Stroke and nonstroke brain attacks in children. Neurology. 2014;82:1434-40.

This paper in a consecutive cohort of children in a tertiary emergency illustrates the importance of stroke mimics in childhood and also compares the data to the adult setting.

22. Braun KP et al. Diagnostic pitfalls in paediatric ischaemic stroke. Dev Med Child Neurol. 2006;48:985-90.

23. Shellhaas RA et al. Mimics of childhood stroke: characteristics of a prospective cohort. Pediatrics. 2006;118:704-9.

24. Harbison $\mathrm{J}$ et al. Rapid ambulance protocol for acute stroke. Lancet. 1999;353:1935.

25. Kleinderdorfer DO et al. Designing a message for public education regarding stroke: does FAST capture enough stroke? Stroke. 2007;10:2864-68.

26. Tong $\mathrm{D}$ et al. Times from symptom onset to hospital arrival in the Get with the Guidelines-Stroke Program 2002 to 2009: temporal trends and implications. Stroke. 2012;43:1912-7.

27. Kothari R et al. Early stroke recognition: developing an out-of-hospital NIH Stroke Scale. Acad Emerg Med. 1997;4:986-90.

28. Purrucker JC et al. Comparison of stroke recognition and stroke severity scores for stroke detection in a single cohort. J Neurol Neurosurg Psychiatry, p. epub ahead of print, December 2014.

29. Nor AM et al. The Recognition of Stroke in the Emergency Room (ROSIER) scale: development and validation of a stroke recognition instrument. Lancet Neurol. 2005;4:727-34.

$30 . \bullet \quad$ Yock-Corrales A et al. Can the FAST and ROSIER adult stroke recognition tools be applied to confirmed childhood arterial ischemic stroke? BMC Pediatr. 2011;11:93.
To our knowledge the first and so far only study analysing the usefulness of stroke recognition tools in children.

31. Mackay MT et al. Childhood posterior circulation arterial ischemic stroke. Stroke. 2010;41:2201-9.

32. Perret E et al. Paediatric posterior circulation arterial ischemic stroke: manifestation, risk factors, neuroimaging and outcome. Swiss Med Wkly. 2011;141:27S.

33. Alberts MJ et al. Recommendations for the establishment of primary stroke centers. Brain Attack Coalition. JAMA. 2000;283:3102-9.

34. Jauch EC et al. Part 11: adult stroke: 2010 American Heart Association guidelines for cardiopulmonary resuscitation and emergency cardiovascular care. Circulation. 2010;122(18 Suppl 3):S818-28.

35. Schroeder EB et al. Determinants of use of emergency medical services in a population with stroke symptoms: the Second Delay in Accessing Stroke Healthcare (DASH II) Study. Stroke. 2000;31:2591-6.

36. Bernard TJ et al. Emergence of the primary pediatric stroke center: impact of the thrombolysis in pediatric stroke trial. Stroke. 2014;45:2018-23.

37. Ichord $\mathrm{RN}$ et al. Interrater reliability of the Pediatric National Institutes of Health Stroke Scale (PedNIHSS) in a multicenter study. Stroke. 2011;42:613-7.

38. Braun KP et al. The course and outcome of unilateral intracranial arteriopathy in 79 children with ischaemic stroke. Brain. 2009;132:544-57.

39. McGlennan $\mathrm{C}$ et al. Delays in investigation and management of acute arterial ischaemic stroke in children. Dev Med Child Neurol. 2008;50(7):537-40.

40. Lasjaunias P, Husson B. Radiological approach to disorders of arterial brain vessels associated with childhood arterial stroke-a comparison between MRA and contrast angiography. Pediatr Radiol. 2004;34:10-5.

41. Jung $S$ et al. Factors that determine penumbral tissue loss in acute ischaemic stroke. Brain.

2013;136(12):3554-60.

42. $\quad$ Stence NV et al. Craniocervical arterial dissection in children: diagnosis and treatment. Curr Treat Options Neurol. 2011;13:636-48.

Recent review on the management and treatment in children with dissection, pinpointing the difficulty in imaging diagnosis and also summarizes to treatment option to the best of our knowledge.

43. Dieleman $\mathrm{N}$ et al. Imaging intracranial vessel wall pathology with magnetic resonance imaging: current prospects and future directions. Circulation. 2014;130:192-201.

44. Bash $\mathrm{S}$ et al. Intracranial vascular stenosis and occlusive disease: evaluation with CT angiography, MR angiography, and digital subtraction angiography. Am J Neuroradiol. 2005;26:1012-21.

45. Miglioretti DL et al. The use of computed tomography in pediatrics and the associated radiation exposure and estimated cancer risk. JAMA Pediatr. 2013;167:700-7.

46. Mathews JD et al. Cancer risk in 680,000 people exposed to computed tomography scans in childhood or adolescence: data linkage study of 11 million Australians. BMJ. 2013;346:f2360. 
47. Sølling C et al. Feasibility and logistics of MRI before thrombolytic treatment. Acta Neurol Scand. 2009; 120:143-9.

In view of our statement that MR imaging should be the primary method of emergency stroke diagnosis in children, this paper helps in setting up a diagnostic chain in emergency to fulfill this demand.

48. Riou EM et al. Cerebrospinal fluid analysis in the diagnosis and treatment of arterial ischemic stroke. Pediatr Neurol. 2008;38:1-9.

49. Benseler S, Pohl D. Childhood central nervous system vasculitis. Handb Clin Neurol. 2013;112:1065-78.

50. Rønning $\mathrm{O}$ et al. Should stroke victims routinely receive supplemental oxygen? A quasi-randomized controlled trial. Stroke. 1999;30:2033-7.

51. Singhal AB et al. A pilot study of normobaric. Stroke. 2005;36:797-802.

52. Leonardi-Bee J et al. Blood pressure and clinical outcomes in the International Stroke Trial. Stroke. 2002;33:1315-20.

53. Ahmed $\mathrm{N}$ et al. Relationship of blood pressure, antihypertensive therapy, and outcome in ischemic stroke treated with intravenous thrombolysis. Retrospective analysis from Safe Implementation of Thrombolysis in Stroke-International Stroke Thrombolysis Register (SITS-IST. Stroke. 2009;40:2442-9.

54. Brush LN et al. Hypertension at time of diagnosis and long-term outcome after childhood ischemic stroke. Neurology. 2013;80(13):1225-30.

55. Willsher A et al. How commonly do children with complex cerebral arteriopathy have renovascular disease? Dev Med Child Neurol. 2013;55:335-40.

56. Prasad Ket al. Fever is associated with doubling of odds of short term mortality in ischemic stroke: an updated metaanalysis. Acta Scand. 2010;122:404-8.

57. den Hertog HM et al. The Paracetamol (Acetominophen) in Stroke (PAIS) trial: a multicentre, randomised placebo controlled, phase III trial. Lancet Neurol. 2009;8:434-40.

58. Baird TA et al. Persistent poststroke hyperglycemia is independently associated with infarct expansion and worse clinical outcome. Stroke. 2003;34:2208-14.

59. Parsons MW et al. Acute hyperglycemia adversely affects stroke outcome: a magnetic resonance imaging and spectroscopy study. Ann Neurol. 2002;52:20-8.

60. Agarwal S et al. Interaction of age with the ischaemic penumbra, leptomeningeal collateral circulation and haemodynamic variables in acute stroke: a pilot study. Neurol Neurosurg Psychiatry. 2013;84(3):271-6.

61. Campbell BC et al. Failure of collateral blood flow is associated with infarction growth in ischemic stroke. J Cereb Blood Flow Metab. 2013;33:1168-72.

62. Goeggel Simonetti B. Basilar artery stroke in childhood. Dev Med Child Neurol. 2013;55:65-70.

63. Mattle HP et al. Comparison of intrarterial and intravenous thrombolysis for ischemic stroke with hyperdense middle cerebral artery sign. Stroke. 2008;39:379-83.
64. Wardlaw JM et al. Thrombolysis for acute ischaemic stroke. Cochrane Syst Rev. 2003;4, CD000213.

65. NINDS group. Tissue plasminogen activator for acute ischemic stroke. N Engl J Med. 1995;333:1581-7.

66. Sen $\mathrm{S}$ et al. IV vs. IA TPA in acute ischemic stroke with CT angiographic evidence of major vessel occlusion: a feasibility study. Neurocrit Care. 2009;11:76-81.

67. Investigators IS. Combined intravenous and intraarterial recanalization for acute ischemic stroke: the Interventional Management of Stroke Study. Stroke. 2004;35:904-11.

68. Sandercock P et al. The benefits and harms of intravenous thrombolysis with recombinant tissue plasminogen activator within $6 \mathrm{~h}$ of acute ischaemic stroke (the third international stroke trial [IST-3]: a randomised controlled trial. Lancet. 2012;379:2352-63.

69. Berkhemer OA et al. A randomized trial of intraarterial treatment for acute ischemic stroke. NEJM. 2015;372:11-20.

70. Arnold $\mathrm{M}$ et al. Thrombolyses in childhood stroke: report of two cases and review of the literature. Stroke. 2009;40(3):801-7.

71. Alshekhlee A et al. Thrombolysis for children with acute ischemic stroke: a perspective from the kids' inpatient database. Pediatr Neurol. 2013;49:313-8.

72. Amlie-Lefond $\mathrm{C}$ et al. Use of alteplase in childhood arterial ischaemic stroke: a multicentre, observational cohort study. Lancet Neurol. 2009;8(6):530-6.

73. Andrew $\mathrm{M}$ et al. Maturation of the hemostatic system during childhood. Blood. 1992;80(8):1998-2005.

74. Monagle P et al. Developmental haemostasis. Impact for clinical haemostasis laboratories. Thromb Haemost. 2006;95(2):362-72.

75. Putaala J et al. Thrombolysis in young adults with ischemic stroke. Stroke. 2009;40:2085-91.

In view of the paper of Bigi et al, the information of this paper is important for our decision taking in acute treamtent of childhood stroke.

76.• Ellis MJ et al. Endovascular therapy in children with acute ischemic stroke: review and recommendations. Neurology. 2012;79:158-64.

In view of the recent results from the Mr CLEAN study an important report and review on endovascular treatment of children with ischaemic stroke - also giving some detailed cautious recommendation how to proceed with children.

77. Berge E et al. Anticoagulants versus antiplatelet agents for acute ischaemic stroke. Cochrane Database Syst Rev. 2002;4, CD003242.

78. Goldenberg NA et al (2009) Antithrombotic treatments, outcomes, and prognostic factors in acute childhood-onset arterial ischaemic stroke: a multicentre, observational, cohort study. Lancet.

79. Strater R et al. Aspirin versus low-dose low-molecularweight heparin: antithrombotic therapy in pediatric. Stroke. 2001;32(11):2554-8.

80. Amlie-Lefond C, Fullerton H. Rashes, sniffles, and stroke: a role for infection in ischemic stroke of childhood. Infect Disord Drug Targets. 2010;10:67-75. 
81. Soman T et al. The risks and safety of clopidogrel in pediatric arterial ischemic stroke. Stroke. 2006;37:1120-2.

82. Gentilomo C et al. The risks and safety of clopidogrel in pediatric arterial ischemic stroke. Pediatr Cardiol. 2011;32:167-75.

83. Kernan $\mathrm{WN}$ et al. Guidelines for the prevention of stroke in patients with stroke and transient ischemic attack: a guideline for healthcare professionals from the American Heart Association/ American Stroke Association. Stroke. 2014;45:160-236.

84. Monagle $\mathrm{P}$ et al. A multicenter, randomized trial comparing heparin/warfarin and acetylsalicylic acid as primary thromboprophylaxis for 2 years after the Fontan procedure in children. J Am Coll Cardiol. 2011;58:645-5.

85. So TY et al. Efficacy, complications and clinical outcome of endovascular treatment for intracranial intradural arterial dissections. Clin Neurol Neurosurg. 2014;117:6-11.

86. Ahlhelm F et al. Endovascular treatment of cervical artery dissection: ten case reports and review of the literature. Intervent Neurol. 2012;1:143-50.

87. Lai YJ et al. Endovascular therapy in pediatric intracranial carotid artery dissection. Pediatr Neurol. 2010;42:291-4.

88. Rahme R et al. Malignant MCA territory infarction in the pediatric population: subgroup analysis of the Greater Cincinnati/Northern Kentucky Stroke Study. Childs Nerv Syst. 2013;29:99-103.

89. Montgomery AK et al. Decompressive craniectomy in childhood posterior circulation stroke: a case series and review of the literature. Pediatr Neurol. 2012;47(3):193.

90. Shah S et al. Decompressive hemicraniectomy in pediatric patients with malignant middle cerebral artery infarction: case series and review of the literature. World Neurosurg. 2013;80:126-33.

Most recent report with an extensive review on the importance of recognition and treatment of malignant infarction.

91. Smith SE et al. Outcome following decompressive craniectomy for malignant middle cerebral artery infarction in children. Dev Med Child Neurol. 2011;53:29-33.
92. Vahedi K et al. Sequential-design, multicenter, randomized, controlled trial of early decompressive craniectomy in malignant middle cerebral artery infarction (DECIMAL Trial). Stroke. 2007;38:2506-17.

93. Jüttler E et al. Decompressive Surgery for the Treatment of Malignant Infarction of the Middle Cerebral Artery (DESTINY): a randomized, controlled trial. Stroke. 2007;38:2518-25.

94. Miravet E et al. Clinical and radiological features of childhood cerebral infarction following varicella zoster virus infection. Dev Med Child Neurol. 2007;49:41722.

95. Askalan R et al. Chickenpox and stroke in childhood: a study of frequency and causation. Stroke. 2001;32:1257-62.

96. Bernard TJ et al. Biomarkers of hypercoagulability and inflammation in childhood-onset arterial ischemic stroke. J Pediatr. 2010;156:651-6.

97. Eleftheriou D et al. Endothelial injury in childhood stroke with cerebral arteriopathy: a cross-sectional study. Neurology. 2012;79:2089-96.

98. Asimos AW, Ward S, Brice JH, Rosamond WD, Goldstein LB, Studnek J. Out-of-hospital stroke screen accuracy in a state with an emergency medical services protocol for routing patients to acute stroke centers. Ann Emerg Med. 2014;64:509-15.

99. Quain DA et al. Improving access to acute stroke therapies: a controlled trial of organised pre-hospital and emergency care. Med J Aust. 2008;189:429-33.

100. Bigi S et al. Acute ischemic stroke in children versus young adults. Ann Neurol. 2011;70:245-54.

Comparison from a paediatric and young adult stroke group, pointing to the importance that childhood compared to young adult stroke does not really differ in severity at manifestation and also outcome. This data supports the importance of emergency treatment for these children.

101. Fuentes B et al. Consistency of the benefits of stroke units over years of operation: an 8-year effectiveness analysis. Cerebrovasc Dis. 2006;21:173-9.

102. Stroke Unit Trialist Collaboration. Cochrane Database Syst Rev. 2013;9:CD000197. doi: 10.1002/ 14651858.CD000197.

103. Seenan P et al. Stroke units in their natural habitat: systematic review of observational studies. Stroke. 2007;38:1886-92. 